\title{
Revisiting conserved charges in higher curvature gravitational theories
}

\author{
M. Ghodrati ${ }^{1, a}$, K. Hajian ${ }^{2, b}$, M. R. Setare ${ }^{3, c}$ \\ ${ }_{1}^{1}$ Michigan Center for Theoretical Physics, Randall Laboratory of Physics, University of Michigan, Ann Arbor, MI 48109-1040, USA \\ ${ }^{2}$ School of Physics, Institute for Research in Fundamental Sciences (IPM), P.O. Box 19395-5531, Tehran, Iran \\ ${ }^{3}$ Department of Science, Campus of Bijar, University of Kurdistan, Bijar, Iran
}

Received: 10 November 2016 / Accepted: 1 December 2016 / Published online: 20 December 2016

(C) The Author(s) 2016. This article is published with open access at Springerlink.com

\begin{abstract}
Restricting the covariant gravitational phase spaces to the manifold of parametrized families of solutions, the mass, angular momenta, entropies, and electric charges can be calculated by a single and simple method. In this method, which has been called the "solution phase space method," conserved charges are unambiguous and regular. Moreover, assuming the generators of the charges to be exact symmetries, entropies and other conserved charges can be calculated on almost arbitrary surfaces, not necessarily horizons or asymptotics. Hence, the first law of thermodynamics would be a local identity relating the exact symmetries to which the mass, angular momentum, electric charge, and entropy are attributed. In this paper, we apply this powerful method to the $f(R)$ gravitational theories accompanied by the terms quadratic in the Riemann and Ricci tensors. Furthermore, conserved charges and the first law of thermodynamics for some of their black hole solutions are exemplified. The examples include warped $\mathrm{AdS}_{3}$, charged static BTZ, and 3-dimensional $z=3$ Lifshitz black holes.
\end{abstract}

\section{Contents}

1 Introduction . . . . . . . . . . . . . 1

2 Solution phase space method . . . . . . . . . 2

2.1 Covariant phase space formulation ...... 3

2.2 Solution phase space method; conserved charges and the first law(s) . . . . . . . . . 5 5

3 Applying the method to higher curvature theories . . 6

4 Some examples . . . . . . . . . . . . . . . 8

5 Conclusion ................. . . 12

References ................ . . 12

a e-mail: ghodrati@umich.edu

b e-mail: kamalhajian@ipm.ir

c e-mail: rezakord@ipm.ir

\section{Introduction}

Since the realization of general relativity and Noether's theorems in 1915, there have been numerous attempts to attribute local (and later quasi-local) conserved charges to the symmetries in the presence of gravity. Nowadays, a century after that, the literature on this subject is rich and well established, but still in progress: local conserved charges have not been consistently formulated, while there are different successful formulations for quasi-local conserved charges (see Refs. $[1,2]$ as reviews). Among the different approaches, two main lines of formulation can be distinguished: one is the Hamiltonian formulation which is based on space + time decomposition, and the other one is the Lagrangian formulation which is based on spacetime covariance. Reviewing the timeline of the major progress in these two formulations (which for sure might miss some interesting contributions) can give us an overview, in addition to clarify the motivations of the analysis in this paper.

Precursor of the Hamiltonian formulation was the introduction of quasi-local charges by Komar [3]. In the Komar's method, quasi-local mass and angular momentum for asymptotic flat solutions could be found by an integration over a codimension-2 surface at constant time asymptotics. Soon after, the Hamiltonian formulation of the gravitational theories was elaborated in a series of works in 1959-1962 by Arnowitt-Deser-Misner [4-6], known as the ADM formulation. Hence, in addition to introducing a sophisticated formulation for gravitational dynamics, the calculation of the mass and angular momentum at the constant time asymptotics was put on a firm basis (reviewed e.g. in Ref. [7]). After that, a similar formulation for the null asymptotics was proposed by Bondi et al. [8,9]. The Hamiltonian formulation for the asymptotic flat spacetimes reached its mature presentation by Regge-Teitelboim [10], emphasizing the role of the surface terms in the Hamiltonian. Nonetheless, there was a 
shortcoming of the formulation, when the asymptotic flatness was relaxed to include asymptotic (anti) de Sitter solutions, mainly because of the appearance of divergent conserved charges. Later progress in this line of formulation has been mainly in the direction of ameliorating this problem (see the review [11]). Transferring to the Hamilton-Jacobi formulation by Brown-York [12], ensued by addition of a surface counterterm to the Lagrangian [13], has been one of them. Reformulation of conserved charges based on covariantly defined conserved currents, and subtracting the contributions from a reference solution, has been another method proposed by Abbott-Deser [14], and this was completed and extended to higher curvature theories by Deser-Tekin $[15,16]$. This method is known as the ADT method in the literature. Last but not least a contribution has been presented by Kim et al. [17], and it is known as the quasi-local method. It is based on the ADT off-shell conserved current, while two major changes are considered: (1) instead of considering the difference between the solution and a reference solution, a oneparameter integration from the reference solution to the solution under consideration is performed (advocated in Refs. $[18,19]),(2)$ the surface of integration is relaxed to be in the interior of the geometry. Hence, it provides a powerful method for calculating conserved charges, specifically for black hole solutions.

The second line of formulating conserved charges is based on the Lagrangian, which is covariant from the beginning. It was initiated by Ashtekar et al. [20,21] and CrnkovicWitten [22], and was consistently formulated in a series of works by Wald et al. [18,23-25]. In this formulation, which is called covariant phase space formulation (see Refs. [2628] for reviews), a covariant phase space was built without a space + time decomposition. The phase space manifold was constructed from dynamical fields all over the spacetime, without recruiting their momentum conjugates. The symplectic form was read from the Lagrangian, which entailed a concrete formulation for conserved charges associated with diffeomorphisms and gauge transformations. Besides, in this progress, the entropy of the non-extermal [24,25] (and later, of the extremal [29]) black holes was introduced as a conserved charge calculated on black hole horizons. Later in 2002, Barnich-Brandt reformulated the formalism in the language of variational bicomplex, in addition to proposing a version directly from the equation of motion (EoM) instead of the Lagrangian [30]. The conceptually strong point (but pragmatically weak point) of the covariant phase space formulation is that the phase space manifold and its tangent space, which are crucial for explicit calculation of conserved charges, are determined by some fall-off conditions. The usual fall-off conditions, although restrict the manifold, do not usually determine it such that calculation of the charges could be performed explicitly. To put the formulation into its full power of calculability, in Ref. [31] the manifold and its tangent space are constructed explicitly and directly from the beginning. The elaboration of the phase space and its tangent space, accompanied by relaxing the calculation of the entropy over horizons, has made the formulation a universal tool in the context of conserved charge calculations. This method can be dubbed a solution phase space method, because the phase space is constructed by some family of parametrized solutions. Interestingly, the recent independent progress, the one by Kim et al. [17] in the Hamiltonian formulation, and the solution phase space method [31] in the Lagrangian formulation, has brought about the two lines of formulations to converge.

In this paper, we apply the solution phase space method to the higher curvature theories. For clarity, we will focus on $f(R)$ gravity accompanied by quadratic terms in the Riemann and Ricci tensors (see Lagrangian (3.1)), although the generalization is straightforward. One of the motivations for this work is examining the method for gravitational theories beyond the Einstein-Hilbert gravity. Another motivation is providing detailed materials needed to perform calculations for general enough higher curvature theories. The analysis can be considered in parallel with higher curvature analysis in other methods, specifically the ADT and quasi-local methods studied e.g. in Refs. [15,16,32-43]. In the following sections, first a review on the solution phase space method is presented. Then it is applied to the higher curvature theories. Finally, some interesting examples are provided and compared with the results of other methods.

\section{Solution phase space method}

Solution phase space method (SPSM) is a method for calculating conserved charges in gravitational theories. The SPSM is based on a powerful but not yet fully appreciated covariant formulation of gravitational phase spaces, which we are going to review in the next subsection. It is applicable to the solutions which are parametrized by some parameters $p_{j}$. Specifically, it is a convenient method for calculating mass, angular momenta, entropies, and electric charges associated with the black hole solutions, although it is not exclusive to them $[26,31]$.

Before delving into the details, it can be helpful to have a look at the big picture and the bottom line: calculation of variation of a conserved charge needs three pieces of information as inputs: (1) the theory in $d$-dimensional spacetime, (2) the solution and some perturbation around it for which charge is calculated, and (3) the symmetry to which the charge is attributed. At the end of the day, integrating a $d-2$-form $\boldsymbol{k}_{\eta}(\hat{\delta} \Phi, \hat{\Phi})$ over any codimension- 2 surface yields the variation of the charge. Concerning the three inputs mentioned above, $\boldsymbol{k}$ is unambiguously determined by the theory. Its arguments $\hat{\Phi}$ and $\hat{\delta} \Phi$ denote some elaborated solutions and per- 
turbations. $\eta$ carries information as regards the symmetry. If the result would be integrable, then an integration over $\hat{\delta} \Phi$ produces the finite charge.

\subsection{Covariant phase space formulation}

Covariant phase space formulation is an appropriate and well-established construction of the gravitational phase spaces [20-25]. To have a self-contained document, we will review the basics of the formulation here, which might have some overlaps with the reviews in Refs. [31,44]. At the outset, it would be useful recalling some relevant elementary properties of a phase space. Phase space $\mathcal{F}(\mathcal{M}, \Omega)$ is a manifold $\mathcal{M}$ equipped with a closed non-degenerate symplectic form $\Omega$. In order to introduce a physical phase space, one usually begins with a given Lagrangian. For example, dynamics of a particle in one dimension can be described by the Lagrangian $L=\frac{m \dot{\mathrm{q}}^{2}}{2}-V(\mathrm{q}) . \mathcal{M}$ would be built by the position and its momentum conjugate $(q, p)$, equipped with the canonical symplectic 2 -form $\Omega=\delta \mathrm{p} \wedge \delta \mathrm{q}$. A simple way to derive this symplectic form is to vary the Lagrangian

$\delta L=\left(\frac{\partial L}{\partial \mathrm{q}}-\frac{\mathrm{d}}{\mathrm{d} t} \frac{\partial L}{\partial \dot{\mathrm{q}}}\right) \delta \mathrm{q}+\frac{\mathrm{d}}{\mathrm{d} t}(\mathrm{p} \delta \mathrm{q})$.

Then, by recognizing the first term as the EoM, and the second term as a total derivative, $\frac{\mathrm{d}}{\mathrm{d} t}$ in the latter has to be dropped, and its exterior derivative on the phase space should be taken:

$$
\frac{\mathrm{d}}{\mathrm{d} t}(\mathrm{p} \delta \mathrm{q}) \rightarrow \mathrm{p} \delta \mathrm{q} \rightarrow \delta(\mathrm{p} \delta \mathrm{q})=\delta \mathrm{p} \wedge \delta \mathrm{q}
$$

Equipped with $\Omega$, and for a given vector field $v$ on the $\mathcal{M}$, a charge variation $\delta H_{v}$ can be defined by

$\delta H_{v} \equiv v \cdot \Omega$.

For instance, in our simple example, choosing $v=\partial_{\mathrm{q}}$, then $\delta H_{v}=\partial_{\mathrm{q}} \cdot \Omega=\delta \mathrm{p}$. Hence, $H_{v}=\mathrm{p}$, which is the mathematical manifestation of "momentum is the charge attributed to the translation in space". Notice that in order for a charge to be conserved, one needs extra conditions. In the case of the simple example mentioned above, $\mathrm{p}$ would be conserved if only $V$ (q) would be a constant.

Covariant phase space: Let us begin with a given Lagrangian in $d$-dimensional spacetime, with some classical dynamical fields, collectively denoted by $\Phi\left(x^{\mu}\right)$. The fields might include the metric $g_{\alpha \beta}$, some Abelian gauge fields $A_{\mu}^{a}$, some scalars $\phi^{I}$, etc. It is usual to build the phase space canonically, i.e. to build the $\mathcal{M}$ from a subset of field configurations $\Phi(\vec{x})$ and their momentum conjugates defined on some privileged time foliation of spacetime. In this construction, solutions to the equation of motion are some curves on $\mathcal{M}$ parametrized by the time. Interestingly, in the context of generally covariant gravitational theories, there is a more suitable construction which does not break general covariance by specifying a time foliation. In this construction, $\mathcal{M}$ is composed of dynamical field configurations all over the spacetime $\Phi\left(x^{\mu}\right)$. On the other hand, the field conjugates would not be needed to construct the manifold. As a result, any solution to the equation of motion in the phase space would be a point on $\mathcal{M}$, instead of a curve. The tangent space of the manifold is also constituted from a subset of perturbations $\delta \Phi\left(x^{\mu}\right)$.

Symplectic structure: The manifold $\mathcal{M}$ which is constructed is a phase space. The symplectic 2-form is constructed from the Lagrangian $d$-form $\mathbf{L}$. To this end, using the same method as in Eqs. (2.1) and (2.2), first the Lee-Wald $(d-1)$-form $\Theta$ is picked up from the surface term appearing in the variation of Lagrangian:

$\delta \mathbf{L}=\mathbf{E}_{\Phi} \delta \Phi+\mathrm{d} \Theta_{\mathrm{LW}}(\delta \Phi, \Phi)$.

In the equation above, $\mathbf{E}_{\Phi}$ denotes equations of motion for the fields $\Phi$, on which the summation convention should be understood. $\delta$ and d are exterior derivatives on $\mathcal{M}$ and on spacetime, respectively. Then the pre-symplectic form can be defined as [23-25]

$\Omega_{\mathrm{LW}}\left(\delta_{1} \Phi, \delta_{2} \Phi, \Phi\right) \equiv \int_{\Sigma} \omega_{\mathrm{LW}}\left(\delta_{1} \Phi, \delta_{2} \Phi, \Phi\right)$

where

$\omega_{\mathrm{LW}}\left(\delta_{1} \Phi, \delta_{2} \Phi, \Phi\right)=\delta_{1} \Theta_{\mathrm{LW}}\left(\delta_{2} \Phi, \Phi\right)-\delta_{2} \Theta_{\mathrm{LW}}\left(\delta_{1} \Phi, \Phi\right)$.

The $\Sigma$ is some codimension-1 (Cauchy) surface and $\delta_{1,2} \Phi$ are some members of the tangent space. The $\omega_{\mathrm{LW}}$, which is a 2 -form over the phase space and a $d-1$-form over the spacetime, is called a pre-symplectic current. By construction, $\Omega$ in Eq. (2.5) is antisymmetric in $\delta_{1} \Phi \leftrightarrow \delta_{2} \Phi$, and is a closed form $\delta \Omega=0$. If it would be a non-degenerate form, it could be used to construct a symplectic structure. In this case, one drops the prefix in "pre-symplectic," and calls it a symplectic form.

Conservation: Apparently, $\Omega_{\mathrm{LW}}$ in Eq. (2.5) depends on a non-covariantly chosen surface $\Sigma$. In order to make $\Omega_{\mathrm{LW}}$ independent of $\Sigma$, which in this context is called "conservation of symplectic form," one needs $\mathrm{d} \omega_{\mathrm{LW}}=0$. Moreover, the flow of $\omega_{\mathrm{LW}}$ passing throughout the boundaries $\partial \Sigma$ should vanish. The former is achieved if $\Phi$ and $\delta \Phi$ satisfy the EoM and the linearized EoM, respectively. So, it is standard to restrict the phase space to the solutions, as we will do in the rest of the paper. On the other hand, achievement of the latter 
needs extra conditions, usually some boundary conditions on the perturbations.

Ambiguities: There are two kinds of ambiguities present in the covariant phase space formulation; one an irrelevant and another one a relevant. The irrelevant one originates from the fact that the formulation is based on Lagrangian formulation, which is itself ambiguous up to a surface term $\mathbf{L} \rightarrow \mathbf{L}+$ $\mathrm{d} \mathcal{K}$. Nonetheless, although it results to $\Theta \rightarrow \Theta+\delta \mathcal{K}$, but $\omega$ remains intact because of $\delta^{2} \mathcal{K}=0$. Another ambiguity, which is the relevant one, is an ambiguity originating from the definition of $\Theta$ in Eq. (2.4); one can add an exact $(d-1)$ form $\mathrm{d} \mathbf{Y}(\delta \Phi, \Phi)$ to $\Theta_{\mathrm{LW}}(\delta \Phi, \Phi)$, i.e.

$$
\Theta_{\mathrm{LW}}(\delta \Phi, \Phi) \rightarrow \Theta(\delta \Phi, \Phi)=\Theta_{\mathrm{LW}}(\delta \Phi, \Phi)+\mathrm{d} \mathbf{Y}(\delta \Phi, \Phi)
$$

This ambiguity entails corresponding ambiguities in $\Omega$ defined above, through

$$
\begin{aligned}
& \omega\left(\delta_{1} \Phi, \delta_{2} \Phi, \Phi\right) \rightarrow \omega\left(\delta_{1} \Phi, \delta_{2} \Phi, \Phi\right)+\mathrm{d}\left(\delta_{2} \mathbf{Y}\left(\delta_{1} \Phi, \Phi\right)\right. \\
& \left.\quad-\delta_{1} \mathbf{Y}\left(\delta_{2} \Phi, \Phi\right)\right) .
\end{aligned}
$$

Conserved charges: Let us consider a vector field $\xi=\xi^{\mu} \partial_{\mu}$ defined over the spacetime, which generates the diffeomorphism $x^{\mu} \rightarrow x^{\mu}-\xi^{\mu}$. In addition, we might have some scalars on the spacetime $\lambda^{a}$, generating the gauge transformations $A_{\mu}^{a} \rightarrow A_{\mu}^{a}+\partial_{\mu} \lambda^{a}$. We can denote the generator of the combination diffeomorphism+gauge transformations by $\epsilon=\left\{\xi, \lambda^{a}\right\}$ such that $\delta_{\epsilon} \Phi \equiv \mathscr{L}_{\xi} \Phi+\delta_{\lambda} a A^{a}$. Being equipped with the symplectic form, and motivated by the definition of charge Eq. (2.3), we might be able to associate a conserved charge (interchangeably called Hamiltonian generator) to the $\epsilon$. To this end, variation of the charge is defined as [23-25,45]

$$
\begin{gathered}
\delta H_{\epsilon}(\Phi) \equiv \int_{\Sigma}\left(\delta^{[\Phi]} \Theta\left(\delta_{\epsilon} \Phi, \Phi\right)-\delta_{\epsilon} \Theta(\delta \Phi, \Phi)\right) \\
=\int_{\Sigma} \mathrm{d} \boldsymbol{k}_{\epsilon}(\delta \Phi, \Phi)=\oint_{\partial \Sigma} \boldsymbol{k}_{\epsilon}(\delta \Phi, \Phi),
\end{gathered}
$$

where the last equation follows from Stokes' theorem. The $\delta^{[\Phi]}$ emphasizes that $\delta$ acts on dynamical fields, not $\epsilon$. In the equation above, the integrand in the first integration has been replaced by an exact ( $d-1)$-form $\mathrm{d} \boldsymbol{k}_{\epsilon}$. This is the fundamental theorem of the covariant phase space formalism, which can be proved using the on-shell conditions [24,25]. The $(d-2)-$ form $\boldsymbol{k}_{\epsilon}$ can be shown to be explicitly (see e.g. Appendix A in Ref. [31] for the detailed derivation)

$\boldsymbol{k}_{\epsilon}(\delta \Phi, \Phi)=\delta \mathbf{Q}_{\epsilon}-\xi \cdot \Theta(\delta \Phi, \Phi)$,

in which $\mathbf{Q}_{\epsilon}$ is the Noether-Wald charge density [24,25], defined by the relation

$\mathrm{d} \mathbf{Q}_{\epsilon} \equiv \Theta\left(\delta_{\epsilon} \Phi, \Phi\right)-\xi \cdot \mathbf{L}$
Hence, by Eq. (2.10), $\boldsymbol{k}_{\epsilon}$ can be found for a given theory straightforwardly. Putting it into Eq. (2.9), the charge variation $\delta H_{\epsilon}(\Phi)$ can be calculated for an arbitrary generator $\epsilon$. Concerning the conservation, by $\mathrm{d} \omega\left(\delta \Phi, \delta_{\epsilon} \Phi, \Phi\right)=$ $\mathrm{d}^{2} \boldsymbol{k}_{\epsilon}(\delta \Phi, \Phi)=0$ there would not be any source or sink in $\Sigma$. But vanishing of the flux/leakage through $\partial \Sigma$ needs to be investigated.

Integrability: $\delta H_{\epsilon}(\Phi)$, which is calculated by the last integral in Eq. (2.9), might corresponds to the variation of a finite conserved charge $H_{\epsilon}$. In order to investigate this finite conserved charge, integrability over the phase space is needed. This condition is basically $\left(\delta_{1} \delta_{2}-\delta_{2} \delta_{1}\right) H_{\epsilon}(\Phi)=0$, in which $\Phi$ s are any field configuration in the presumed phase space $\mathcal{F}$, and $\delta_{1,2} \Phi$ are any arbitrary chosen member of its tangent space. It follows that this condition can be explained as $[18,23,45]$

$$
\begin{gathered}
\oint_{\partial \Sigma}\left(\xi \cdot \omega\left(\delta_{1} \Phi, \delta_{2} \Phi, \Phi\right)+\boldsymbol{k}_{\delta_{1} \epsilon}\left(\delta_{2} \Phi, \Phi\right)\right. \\
\left.-\boldsymbol{k}_{\delta_{2} \epsilon}\left(\delta_{1} \Phi, \Phi\right)\right)=0, \forall \Phi, \delta_{1,2} \Phi .
\end{gathered}
$$

Symplectic symmetries: As far as conserved charges are concerned, conservation of $\delta H_{\epsilon}$ can be guaranteed if $\epsilon$ is chosen such that

$\omega\left(\delta \Phi, \delta_{\epsilon} \Phi, \Phi\right)=0$

on-shell. It is because there would not be any flow out of the boundaries locally, and hence globally. The family of $\epsilon$ 's with this property, which has been dubbed "symplectic symmetry generators" [46], can be divided into two sets [31]:

1. Non-exact symplectic symmetries The $\epsilon$ for which $\delta_{\epsilon} \Phi \neq$ 0 at least on one point of the phase space. They constitute a closed algebraic structure, and have been proposed to be responsible for generating the phase space of a solution at some given constant thermodynamical variables [45-47]. Hence, by studying them and the phase space generated via exponentiating them, one might hope to understand the microstates of the system.

2. Exact symplectic symmetries The ones for which $\delta_{\epsilon} \Phi=$ 0 all over the phase space. For clarity, let us denote such generators by $\eta=\left\{\zeta, \lambda^{a}\right\}$. They are considered as generators which by their conserved charges, the set of solutions in different thermodynamical variables can be labeled [31]. The phase space constructed by such field configurations has been called "solution phase space," [31] which will be described in a moment.

It has been conjectured that the phase space associated with the geometries without propagating degrees of freedom are 
direct product of these two families of phase spaces: the statistical phase space $\otimes$ the solution phase space $[31,48]$.

\subsection{Solution phase space method; conserved charges and the first law(s)}

In the covariant phase space formulation reviewed above, it is standard to identify the phase space manifold by some asymptotic behaviors, usually through requiring some falloff conditions. Fall-off conditions, although they delimit the phase space manifold, usually do not determine it completely. "Solution phase space method" is an alternative method for determining the phase space manifold. Restricting the covariant phase space formulation to some explicitly identified manifolds, empowers the calculability of this formulation. The specification is in three aspects, which will be described immediately:

1. Identifying the phase space manifold explicitly,

2. Crystallizing the tangent space of the specified manifolds,

3. Concentrating on the exact sympltectic symmetries of the proposed phase space.

Consider a family of (black hole) solutions to a generally covariant gravitational theory. Usually, such a family is identified by some isometries and some parameters $p_{j}$. The field configuration of such a family can be denoted collectively by $\hat{\Phi}\left(x^{\mu}, p_{j}\right)$. The parameters are some arbitrary (but maybe in some restricted domain of) real numbers appearing in the field configuration of the mentioned solutions. The $p_{j}$ can be reparametrized, but they cannot be removed by coordinate transformations. The manifold $\hat{\mathcal{M}}$ can be chosen to be composed of the members of the family, up to unphysical coordinate/gauge transformations. As an example, the set of all Schwarzschild black holes

$\mathrm{d} s^{2}=-\left(1-\frac{2 G m}{r}\right) \mathrm{d} t^{2}+\frac{\mathrm{d} r^{2}}{1-\frac{2 G m}{r}}+r^{2} \mathrm{~d} \theta^{2}+r^{2} \sin ^{2} \theta \mathrm{d} \varphi^{2}$,

parametrized by one free parameter $p_{1}=m \geq 0$, construct a manifold $\hat{\mathcal{M}}$.

The symplectic 2 -form $\hat{\Omega}$ would be simply the Lee-Wald symplectic form (2.5), which is confined to $\hat{\mathcal{M}}$. Therefore, the $\hat{\mathcal{F}}=(\hat{\mathcal{M}}, \hat{\Omega})$ would constitute a phase space, the "solution phase space". Hence, any point of the manifold can be identified by $\hat{\Phi}\left(x^{\mu}, p_{j}\right)$. The tangent space of $\hat{\mathcal{M}}$ is spanned (up to infinitesimal pure gauge transformations) by "parametric variations," which can be found simply by [49]

$\hat{\delta} \Phi=\frac{\partial \hat{\Phi}}{\partial p_{j}} \delta p_{j}$
These variations, which are infinitesimal difference of two solutions, satisfy linearized equation of motion. As a result, they respect $\mathrm{d} \omega_{\mathrm{LW}}\left(\hat{\delta}_{1} \Phi, \hat{\delta}_{2} \Phi, \hat{\Phi}\right)=0$.

In SPSM, the diff+gauge transformations, for which charges are calculated, should be restricted to the symplectic symmetries. However, our main focus would be on the set of exact symmetries. Denoting the generator of the exact symmetries by $\eta=\left\{\zeta, \lambda^{a}\right\}$ such that $\delta_{\eta} \hat{\Phi}=0$, the $\zeta$ would be a Killing vector of all points of the phase space $\hat{\mathcal{M}}$. Besides, its action on the gauge fields has to be canceled by the action of $\lambda^{a}$ 's, i.e. $\mathcal{L}_{\zeta} A_{\mu}^{a}+\partial_{\mu} \lambda^{a}=0$. As it was advertised in Sect. 2.2, conservation of $\hat{\delta} H_{\eta}$ is guaranteed. This is because of the relation $\omega_{\mathrm{LW}}\left(\hat{\delta} \Phi, \delta_{\eta} \hat{\Phi}, \hat{\Phi}\right)=0$, which itself is a result of linearity of $\omega_{\mathrm{LW}}$ in $\delta_{\eta} \hat{\Phi}=0$. Hence, there would not be any local and, therefore, any global flow of $\omega_{\mathrm{LW}}$ out of the boundaries $\partial \Sigma$.

Along with guaranteeing the conservation, focusing on the exact symmetries provides us some other nice features:

- Independence of $\hat{\delta} H_{\eta}$ from the choice of $\partial \Sigma$ : The relation $\omega_{\mathrm{LW}}\left(\hat{\delta} \Phi, \delta_{\eta} \hat{\Phi}, \hat{\Phi}\right)=0$ yields an interesting result: $\hat{\delta} H_{\eta}$ would be independent of the chosen $\partial \Sigma$. It is because of vanishing of $\omega_{\mathrm{LW}}$ all over the $\Sigma$, and hence, vanishing of $\omega_{\mathrm{LW}}$ in the region enclosed between two different integrating surfaces $\partial \Sigma_{1}$ and $\partial \Sigma_{2}$. Then, by the Stokes theorem, and taking the result of Eq. (2.9) into account, the claim is proved. Explaining this result in another way, although the integration in calculating $\hat{\delta} H_{\eta}$ is over a codimension-2 surface $\partial \Sigma$, but the result would be independent of all coordinates, including the two coordinates which are not integrated over.

- Discarding the ambiguity $\mathbf{Y}$ : This is because of $\delta \mathbf{Y}\left(\delta_{\eta} \Phi, \Phi\right)-\delta_{\eta} \mathbf{Y}(\delta \Phi, \Phi)=0$, which is a result of the linearity of the left hand side in $\delta_{\eta} \Phi=0$. Using this identity together with Eq. (2.8) in the definition of charge variations Eq. (2.9), then there would not be any ambiguity in the calculated conserved charges as far as exact symmetries are considered.

Summarizing the last two paragraphs, the charges associated with exact symmetries are conserved, unambiguous, and independent of the chosen closed surfaces of integration $\partial \Sigma$.

So far, the SPSM has provided all materials needed to calculate conserved charge variations. The final tasks would be checking integrability over $\hat{\mathcal{M}}$, and (if integrable) performing the integration. The integrability can be assessed by replacing $\Phi, \delta \Phi$, and $\epsilon$ in the integrability condition Eq. (2.12) by $\hat{\Phi}, \hat{\delta} \Phi$, and $\eta$, respectively. If integrable, then the integration over arbitrary path on $\hat{\mathcal{M}}$ connecting a reference field configuration $\hat{\Phi}\left(\bar{p}_{j}\right)$ to the solution under consideration $\hat{\Phi}\left(p_{j}^{0}\right)$ yields the final result 
$H_{\eta}\left[\hat{\Phi}\left(p^{0}\right)\right]-H_{\eta}[\hat{\Phi}(\bar{p})]=\int_{\bar{p}}^{p^{0}} \hat{\delta} H_{\eta}$.

$H_{\eta}[\hat{\Phi}(\bar{p})]$ is the reference point (i.e. constant of integration) for $H_{\eta}$ defined on some specific reference field configuration $\hat{\Phi}\left(x^{\mu} ; \bar{p}_{j}\right)$.

It is worth mentioning that in order to perform the final tasks mentioned above, there is a shortcut: by the conservation+independence from $\partial \Sigma, \hat{\delta} H_{\eta}$ would only be a function of $p_{j}$ and $\delta p_{j}$, not any coordinate of the spacetime. Hence, one can simply check whether it is a total derivative or not. Then, in the case of a total derivative, the integration can be done by an appropriate choice of a reference field. For instance, if $\hat{\mathcal{M}}$ is parametrized by two parameters $\left\{p_{1}, p_{2}\right\}$, and one has found $\hat{\delta} H_{\eta}=p_{1} \delta p_{2}+p_{2} \delta p_{1}$, then it is a total derivative $\hat{\delta} H_{\eta}=\hat{\delta}\left(p_{1} p_{2}\right)$. So, the integrated charge would be simply $H_{\eta}=p_{1} p_{2}+$ const., where the constant would be fixed by the choice of a reference field configuration.

Before moving on to the next sections, which will provide us explicit examples, it can be useful recalling some remarks in the context of SPSM.

- Not all exact symmetries have integrable conserved charges. Hence, integrability puts constraint on the choice of exact symmetries to which mass, angular momenta etc. are attributed.

- Electric charge associated to the gauge field $A^{a}$, denoted by $Q_{a}$, is the conserved charge of the global gauge transformation $\eta_{Q_{a}}=\left\{0,1^{a}\right\}$ in which $1^{a}$ means $\lambda^{a}=1$ and $\lambda^{b}=0$ for $b \neq a$.

- Similar to the electric charge, mass and angular momenta are conserved charges which are attributed to the geometry as a whole. For the stationary solutions with some number of axial $U(1)$ isometries (labeled by $i$ ), one can choose the coordinates such that the corresponding Killing vectors would be $\partial_{t}$ and $\partial_{\varphi^{i}}$, respectively. Then, up to a conventional normalization, the exact symmetries to which the mass $M$ and angular momenta $J_{i}$ are attributed would be $\eta_{M}=\left\{\partial_{t}+\Omega_{\infty}^{i} \partial_{\varphi^{i}},-\Phi_{\infty}^{a}\right\}$ and $\eta_{J_{i}}=\left\{-\partial_{\varphi^{i}}, 0\right\} . \Omega_{\infty}^{i}$ and $\Phi_{\infty}^{a}$ are asymptotic angular velocities and electric potentials, which are usually adopted to be zero.

- In contrast with the charges mentioned above, to each one of the horizons in a geometry, one can associate an entropy. Hence, there might be more than one entropy in a single geometry, e.g. the entropy of inner, outer or cosmological horizons. Entropies are considered to be conserved charges for the exact symmetries $\eta_{\mathrm{H}}=$ $\frac{2 \pi}{\kappa_{\mathrm{H}}}\left\{\zeta_{\mathrm{H}},-\Phi_{\mathrm{H}}^{a}\right\}$, in which $\kappa_{\mathrm{H}}, \zeta_{\mathrm{H}}, \Phi_{\mathrm{H}}^{a}$ are surface gravity, Killing vector, and electric potential of the horizon, respectively. Notice that the $\zeta_{\mathrm{H}}$ should be accompanied by the rigid gauge transformations $\lambda^{a}=-\Phi_{\mathrm{H}}^{a}$, and be normalized by the surface gravity, in order to have an integrable charge.

- It is worth emphasizing again that assuming the generators of mass, angular momenta, electric charges and entropies to be exact symmetries, these charges can be calculated by integrations over almost arbitrary $\partial \Sigma$, and not necessarily the horizons or asymptotics. In this respect, the entropies are on equal footing with other conserved charges.

First law(s) of thermodynamics: To each one of the horizons denoted by " $\mathrm{H}$ ", an entropy $S_{\mathrm{H}}$, temperature $T_{\mathrm{H}}=\frac{\kappa_{\mathrm{H}}}{2 \pi}$, and some chemical potentials $\Omega_{\mathrm{H}}^{i}, \Phi_{\mathrm{H}}^{a}$ etc. can be attributed [50-52]. The first law of thermodynamics for the chosen horizon relates $\delta S_{\mathrm{H}}$ to the variations of other conserved charges attributed to the whole geometry. In the SPSM, derivation of the first law(s) is very simple, and originates from a local identity; $\eta_{\mathrm{H}}$ is a linear combination of the generators of mass, angular momenta, and electric charges. From this identity, the first law follows by the linearity of the generic charge variations $\delta H_{\epsilon}$ in terms of the generator $\epsilon$ (see Eq. (2.9)). Mathematically [24,25,31],

$$
\begin{aligned}
\eta_{\mathrm{H}} & =\frac{1}{T_{\mathrm{H}}}\left(\eta_{M}-\left(\Omega_{\mathrm{H}}^{i}-\Omega_{\infty}^{i}\right) \eta_{J_{i}}-\left(\Phi_{\mathrm{H}}^{a}-\Phi_{\infty}^{a}\right) \eta_{Q_{a}}\right) \\
\Rightarrow & \delta S_{\mathrm{H}}=\frac{1}{T_{\mathrm{H}}}\left(\delta M-\left(\Omega_{\mathrm{H}}^{i}-\Omega_{\infty}^{i}\right) \delta J_{i}-\left(\Phi_{\mathrm{H}}^{a}-\Phi_{\infty}^{a}\right) \delta Q_{a}\right)
\end{aligned}
$$

where $\delta S_{\mathrm{H}} \equiv \delta H_{\eta_{\mathrm{H}}}, \delta M \equiv \delta H_{\eta_{M}}, \delta J_{i} \equiv \delta H_{\eta_{J_{i}}}$ and $\delta Q_{a} \equiv$ $\delta H_{\eta_{Q a}}$. Notice that the $\delta$ in the proof is a generic perturbation which satisfies linearized EoM. So, it is not restricted to the parametric variations. Moreover, integration over horizons or asymptotics does not play any role in this proof.

\section{Applying the method to higher curvature theories}

SPSM has reproduced successfully conserved charges and first law(s) for the standard (black hole) solutions to EinsteinHilbert gravitational theories. Explicit examples can be found in Refs. [26,31,44]. The goal of this section is utilizing the SPSM for the gravitational theories with higher curvature terms. Explicitly, the Lagrangian which we will focus on, has the metric $g_{\alpha \beta}$, some gauge fields $A_{\mu}^{a}$, and some scalar fields $\phi^{I}$, in arbitrary dimension $d$ :

$$
\begin{aligned}
\mathcal{L}= & \frac{1}{16 \pi G}\left(f(R, \phi)+\mathrm{a}(\phi) R_{\mu \nu} R^{\mu \nu}+\mathrm{b}(\phi) R_{\mu \nu \alpha \beta} R^{\mu \nu \alpha \beta}\right. \\
& \left.-\mathrm{c}_{a b}(\phi) F_{\mu \nu}^{a} F^{b \mu \nu}-2 \mathrm{~d}_{I J}(\phi) \nabla^{\mu} \phi^{I} \nabla_{\mu} \phi^{J}\right) .
\end{aligned}
$$

$R_{\nu \alpha \beta}^{\mu}, R_{\mu \nu}$, and $R$ are Riemann tensor, Ricci tensor, and Ricci scalar, respectively. The $F^{a}=\mathrm{d} A^{a}$ are the field strengths. 
The coefficients $\mathrm{a}(\phi), \mathrm{b}(\phi), \mathrm{c}_{a b}(\phi)$, and $\mathrm{d}_{I J}(\phi)$ are some functions of $\phi^{I}$. Notice that $f(R, \phi)$ covers the EinsteinHilbert gravity with a cosmological constant. Besides, the Gauss-Bonnet theory of gravity is also covered by the Lagrangian (3.1), hence the simplest Lanczos-Lovelock theories are also included [53-56]. Generalization to higher Lanczos-Lovelock theories is straightforward, and we will not consider in this paper. The Lagrangian $d$-form is the Hodge dual of (3.1), $\mathbf{L}=\star \mathcal{L}$,

$\mathbf{L}=\frac{\sqrt{-g}}{d !} \epsilon_{\mu_{1} \mu_{2} \cdots \mu_{d}} \mathcal{L} \mathrm{d} x^{\mu_{1}} \wedge \mathrm{d} x^{\mu_{2}} \wedge \cdots \wedge \mathrm{d} x^{\mu_{d}}$.

$\epsilon_{\mu_{1} \mu_{2} \cdots \mu_{d}}$ is the Levi-Civita symbol, i.e. $\epsilon_{012 \cdots d-1}=+1$ and its sign changes with the odd permutations of the indices. We will use the conventions

$h^{\mu \nu} \equiv \delta g^{\mu \nu}=g^{\mu \alpha} g^{\nu \beta} \delta g_{\alpha \beta}$,

$\delta F^{\mu \nu} \equiv g^{\mu \alpha} g^{\nu \beta}(\delta \mathrm{d} A)_{\alpha \beta}=g^{\mu \alpha} g^{\nu \beta}(\mathrm{d} \delta A)_{\alpha \beta}$.

Hence, the indices for the perturbed fields can be raised and lowered similar to other tensors. Let us label the terms in the Lagrangian (3.1) by $f, \mathrm{a}, \mathrm{b}, \mathrm{c}$, and d, respectively. The EoM for the chosen Lagrangian, considering variations with respect to the metric, gauge fields, and scalar fields, are, respectively [57],

$$
\begin{aligned}
& E_{f \mu \nu}+E_{a \mu \nu}+E_{b \mu \nu}+E_{c \mu \nu}+E_{\mathrm{d} \mu \nu}=0 \\
& E_{f \mu \nu}=\frac{1}{2} f g_{\mu \nu}-f^{\prime} R_{\mu \nu}+\nabla_{\mu} \nabla_{\nu} f^{\prime}-\square f^{\prime} g_{\mu \nu} \\
& E_{\mathrm{a} \mu \nu}=\mathrm{a}\left(\frac{1}{2} R_{\alpha \beta} R^{\alpha \beta} g_{\mu \nu}+\nabla^{\alpha}\left(\nabla_{\mu} R_{\alpha \nu}\right.\right. \\
& \left.\left.\quad+\nabla_{\nu} R_{\alpha \mu}\right)-\nabla_{\alpha} \nabla_{\beta} R^{\alpha \beta} g_{\mu \nu}-\square R_{\mu \nu}-2 R_{\mu \alpha} R_{\nu}^{\alpha}\right) \\
& E_{\mathrm{b} \mu \nu}=\mathrm{b}\left(\frac{1}{2} R_{\rho \sigma \alpha \beta} R^{\rho \sigma \alpha \beta} g_{\mu \nu}\right. \\
& \left.\quad-2 R_{\mu \nu \alpha \beta} R_{\nu}^{\gamma \alpha \beta}-2 \nabla^{\alpha} \nabla^{\beta}\left(R_{\mu \alpha \nu \beta}+R_{\nu \alpha \mu \beta}\right)\right) \\
& E_{\mathrm{c} \mu \nu}=2 \mathrm{c}_{a b}\left(F_{\mu \alpha}^{a} F_{\nu}^{b \alpha}-\frac{1}{4} F_{\alpha \beta}^{a} F^{b \alpha \beta} g_{\mu \nu}\right) \\
& E_{\mathrm{d} \mu \nu}=2 \mathrm{~d}_{I J}\left(\nabla_{\mu} \phi^{I} \nabla_{\nu} \phi^{J}-\frac{1}{2} \nabla^{\alpha} \phi^{I} \nabla_{\alpha} \phi^{J} g_{\mu \nu}\right) \\
& \nabla_{\nu}\left(\mathrm{c}_{a b} F^{b \mu \nu}\right)=0, \\
& 4 \nabla_{\alpha}\left(\mathrm{d}_{I J} \nabla^{\alpha} \phi^{J}\right)+\frac{\partial f}{\partial \phi^{I}}+\frac{\partial \mathrm{a}}{\partial \phi^{I}} R_{\mu \nu} R^{\mu \nu}+\frac{\partial \mathrm{b}}{\partial \phi^{I}} R_{\mu \nu \alpha \beta} R^{\mu \nu \alpha \beta} \\
& \quad-\frac{\partial \mathrm{c}_{a b}}{\partial \phi^{I}} F_{\mu \nu}^{a} F^{b \mu \nu}-2 \frac{\partial \mathrm{d}_{J K}}{\partial \phi^{I}} \nabla^{\mu} \phi^{J} \nabla_{\mu} \phi^{K}=0
\end{aligned}
$$

where the notation $f^{\prime} \equiv \frac{\partial f}{\partial R}$ is used. We need to find $\Theta_{\mathrm{LW}}, \mathbf{Q}_{\epsilon}$, and most importantly, the $\boldsymbol{k}_{\epsilon}$ for this theory. Their derivation and final results are standard practices in the literature. Hence we only report the final results here. Detailed analysis are similar to the simple Einstein-Hilbert Lagrangian, which can be found e.g. in Appendix A of Ref. [44].

By variation of Lagrangian $\delta \mathbf{L}$ and imposing the EoM, the surface $d-1$-form $\Theta_{\mathrm{LW}}$ can be read through Eq. (2.4) to be $\Theta_{\mathrm{LW}}=\star \Theta$, i.e.

$$
\begin{aligned}
\Theta_{\mathrm{LW}}= & \frac{\sqrt{-g}}{(d-1) !} \epsilon_{\mu \mu_{1} \cdots \mu_{d-1}}\left(\Theta_{f}^{\mu}+\Theta_{\mathrm{a}}^{\mu}+\Theta_{\mathrm{b}}^{\mu}+\Theta_{\mathrm{c}}^{\mu}+\Theta_{\mathrm{d}}^{\mu}\right) \\
& \times \mathrm{d} x^{\mu_{1}} \wedge \cdots \wedge \mathrm{d} x^{\mu_{d-1}}
\end{aligned}
$$

in which

$\Theta_{f}^{\mu}(\delta \Phi, \Phi)=\frac{1}{16 \pi G}\left(f^{\prime}\left(\nabla_{\alpha} h^{\mu \alpha}-\nabla^{\mu} h\right)-\nabla_{\alpha} f^{\prime} h^{\mu \alpha}+\nabla^{\mu} f^{\prime} h\right)$,

$\Theta_{\mathrm{a}}^{\mu}(\delta \Phi, \Phi)=\frac{\mathrm{a}}{16 \pi G}\left(2 R_{\alpha \beta} \nabla^{\alpha} h^{\beta \mu}\right.$

$\left.-2 \nabla_{\alpha} R_{\beta}^{\mu} h^{\alpha \beta}-R_{\alpha}^{\mu} \nabla^{\alpha} h+\nabla^{\alpha} R_{\alpha}^{\mu} h-R_{\alpha \beta} \nabla^{\mu} h^{\alpha \beta}+\nabla^{\mu} R_{\alpha \beta} h^{\alpha \beta}\right)$,

$\Theta_{\mathrm{b}}^{\mu}(\delta \Phi, \Phi)=\frac{\mathrm{b}}{4 \pi G}\left(\nabla^{\nu} R_{\alpha \nu \beta}^{\mu} h^{\alpha \beta}-R_{\alpha \nu \beta}^{\mu} \nabla^{\nu} h^{\alpha \beta}\right)$,

$\Theta_{\mathrm{c}}^{\mu}(\delta \Phi, \Phi)=\frac{-1}{4 \pi G} \mathrm{c}_{a b} F^{a \mu \nu} \delta A_{v}^{b}$,

$\Theta_{\mathrm{d}}^{\mu}(\delta \Phi, \Phi)=\frac{-1}{4 \pi G} \mathrm{~d}_{I J} \nabla^{\mu} \phi^{I} \delta \phi^{J}$,

where $h \equiv h_{\alpha}^{\alpha}$. Having the $\Theta$ in our hand, for a generic $\epsilon=\left\{\xi, \lambda^{a}\right\}$, the Noether-Wald $d-2$-form $\mathbf{Q}_{\epsilon}$ can be read through Eq. (2.11) and imposing the EoM, Eq. (3.4), as

$$
\begin{gathered}
\mathbf{Q}_{\epsilon}=\frac{\sqrt{-g}}{(d-2) ! 2 !} \epsilon_{\mu \nu \mu_{1} \cdots \mu_{d-2}}\left(\mathrm{Q}_{f \epsilon}^{\mu \nu}\right. \\
\left.+\mathrm{Q}_{\mathrm{a} \epsilon}^{\mu \nu}+\mathrm{Q}_{\mathrm{b} \epsilon}^{\mu \nu}+\mathrm{Q}_{\mathrm{c} \epsilon}^{\mu \nu}+\mathrm{Q}_{\mathrm{d} \epsilon}^{\mu \nu}\right) \\
\mathrm{d} x^{\mu_{1}} \wedge \cdots \wedge \mathrm{d} x^{\mu_{d-2}},
\end{gathered}
$$

in which

$\mathrm{Q}_{f \epsilon}^{\mu \nu}=\frac{1}{16 \pi G}\left(2 \nabla^{\mu} f^{\prime} \xi^{\nu}-f^{\prime} \nabla^{\mu} \xi^{\nu}\right)-[\mu \leftrightarrow \nu]$,

$\mathrm{Q}_{\mathrm{a} \epsilon}^{\mu \nu}=\frac{\mathrm{a}}{8 \pi G}\left(\nabla^{\mu} R_{\alpha}^{\nu} \xi^{\alpha}+R_{\alpha}^{\nu} \nabla^{\alpha} \xi^{\mu}-\nabla^{\alpha} R_{\alpha}^{\nu} \xi^{\mu}\right)-[\mu \leftrightarrow \nu]$,

$\mathrm{Q}_{\mathrm{b} \epsilon}^{\mu \nu}=\frac{\mathrm{b}}{4 \pi G}\left(\nabla^{\alpha} R_{\alpha \beta}^{\mu \nu} \xi^{\beta}-R^{\mu \alpha \nu \beta} \nabla_{\alpha} \xi_{\beta}\right)-[\mu \leftrightarrow \nu]$,

$\mathrm{Q}_{\mathrm{c} \epsilon}^{\mu \nu}=\frac{-1}{4 \pi G} \mathrm{c}_{a b} F^{a \mu \nu}\left(A_{\rho}^{b} \xi^{\rho}+\lambda^{b}\right)$,

$\mathrm{Q}_{\mathrm{d} \epsilon}^{\mu \nu}=0$.

After varying $\mathbf{Q}_{\epsilon}$ with respect to all dynamical fields and utilizing the textbook relations

$\delta \sqrt{-g}=\frac{\sqrt{-g}}{2} h_{\alpha}^{\alpha}$,

$\delta \Gamma_{\mu \nu}^{\lambda}=\frac{1}{2} g^{\lambda \sigma}\left(\nabla_{\mu} h_{\sigma \nu}+\nabla_{\nu} h_{\sigma \mu}-\nabla_{\sigma} h_{\mu \nu}\right), \delta \epsilon_{\mu \nu \mu_{1} \cdots \mu_{d-2}}=0$,

$\delta R_{\mu \nu \alpha \beta}=\frac{1}{2}\left(2 R_{\mu \nu \alpha \gamma} h_{\beta}^{\gamma}-\nabla_{\mu} \nabla_{\alpha} h_{\beta \nu}+\nabla_{\mu} \nabla_{\beta} h_{\alpha \nu}\right.$

$\left.-\nabla_{\mu} \nabla_{\nu} h_{\alpha \beta}+\nabla_{\nu} \nabla_{\alpha} h_{\beta \mu}-\nabla_{\nu} \nabla_{\beta} h_{\alpha \mu}+\nabla_{\nu} \nabla_{\mu} h_{\alpha \beta}\right)$,

$\delta R_{\mu \nu}=\frac{1}{2}\left(\nabla_{\alpha} \nabla_{\mu} h^{\alpha}{ }_{\nu}+\nabla_{\alpha} \nabla_{\nu} h_{\mu}^{\alpha}-\square h_{\mu \nu}-\nabla_{\mu} \nabla_{\nu} h\right)$,

$\delta R=\nabla_{\mu} \nabla_{\nu} h^{\mu \nu}-\square h-R_{\mu \nu} h^{\mu \nu}$, 
one can find $\boldsymbol{k}_{\epsilon}$ by Eq. (2.10), to calculate variations of the conserved charges. The result, the final applicable tensor for calculation of the conserved charges turns out to be

$$
\begin{aligned}
\boldsymbol{k}_{\epsilon}(\delta \Phi, \Phi)= & \frac{\sqrt{-g}}{(d-2) ! 2 !} \epsilon_{\mu \nu \mu_{1} \cdots \mu_{d-2}}\left(k_{f \epsilon}^{\mu \nu}+k_{\mathrm{a} \epsilon}^{\mu \nu}+k_{\mathrm{b} \epsilon}^{\mu \nu}\right. \\
& \left.+k_{\mathrm{c} \epsilon}^{\mu \nu}+k_{\mathrm{d} \epsilon}^{\mu \nu}\right) \mathrm{d} x^{\mu_{1}} \wedge \cdots \wedge \mathrm{d} x^{\mu_{d-2}}
\end{aligned}
$$

where, using the notation $f^{\prime} \equiv \frac{\partial f}{\partial R}$,

$$
\begin{aligned}
& k_{f \epsilon}^{\mu \nu}(\delta \Phi, \Phi)=\frac{1}{16 \pi G}\left[\left(h^{\mu \alpha} \nabla_{\alpha} \xi^{\nu}-\nabla^{\mu} h^{\nu \alpha} \xi_{\alpha}\right.\right. \\
& \left.-\frac{1}{2} h \nabla^{\mu} \xi^{\nu}\right) f^{\prime}+2\left(R^{\mu \alpha} \nabla_{\alpha} h-\nabla_{\alpha} R h^{\mu \alpha}-R_{\alpha}^{\mu} \nabla_{\beta} h^{\alpha \beta}\right. \\
& \left.-\square \nabla^{\mu} h+\nabla_{\alpha} \nabla^{\mu} \nabla_{\beta} h^{\alpha \beta}-\nabla^{\mu}\left(R_{\alpha \beta} h^{\alpha \beta}\right)+\frac{1}{2} \nabla^{\mu} R h\right) \xi^{\nu} f^{\prime \prime} \\
& +2\left(\nabla^{\mu} \delta \phi^{I}-h_{\alpha}^{\mu} \nabla^{\alpha} \phi^{I}+\frac{1}{2} h \nabla^{u} \phi^{I}\right) \xi^{\nu} \frac{\partial f^{\prime}}{\partial \phi^{I}}-\delta \phi^{I} \nabla^{\mu} \xi^{\nu} \frac{\partial f^{\prime}}{\partial \phi^{I}} \\
& +\left(R_{\alpha \beta} h^{\alpha \beta}-\nabla_{\alpha} \nabla_{\beta} h^{\alpha \beta}+\square h\right)\left(\nabla^{\mu} \xi^{\nu} f^{\prime \prime}-2 \nabla^{\mu} R \xi^{\nu} f^{\prime \prime \prime}\right. \\
& \left.-2 \nabla^{\mu} \phi^{I} \xi^{\nu} \frac{\partial f^{\prime \prime}}{\partial \phi^{I}}\right)+2 \delta \phi^{I} \nabla^{\mu} \phi^{J} \xi^{\nu} \frac{\partial^{2} f^{\prime}}{\partial \phi^{I} \partial \phi^{J}} \\
& \left.+2 \delta \phi^{I} \nabla^{\mu} R \xi^{\nu} \frac{\partial f^{\prime \prime}}{\partial \phi^{I}}\right]-\Theta_{f}^{\mu} \xi^{\nu}-[\mu \leftrightarrow \nu],
\end{aligned}
$$$$
k_{\mathrm{a} \epsilon}^{\mu \nu}(\delta \Phi, \Phi)=\frac{\mathrm{a}}{16 \pi G}\left[\left(\nabla^{\alpha} R_{\alpha}^{\mu} h-\nabla_{\alpha} R h^{\mu \alpha}-\nabla^{\mu}\left(R_{\alpha \beta} h^{\alpha \beta}\right)\right.\right.
$$$$
\left.+\nabla^{\mu} \nabla_{\alpha} \nabla_{\beta} h^{\alpha \beta}-\nabla^{\mu} \square h\right) \xi^{\nu}+\left(2 \nabla_{\beta} R_{\alpha}^{\mu} h^{\beta v}\right.
$$$$
-2 R^{\mu \beta} \nabla_{\beta} h_{\alpha}^{\nu}-2 \nabla^{\mu} R_{\alpha \beta} h^{\nu \beta}-\nabla^{\mu}\left(\nabla_{\alpha} \nabla^{\nu} h-\nabla_{\beta} \nabla_{\alpha} h^{\nu \beta}\right.
$$$$
\left.\left.+\square h_{\alpha}^{v}-\nabla^{\beta} \nabla^{v} h_{\alpha \beta}\right)+\nabla^{\mu} R_{\alpha}^{v} h+2 R^{\mu \beta} \nabla^{v} h_{\alpha \beta}\right) \xi^{\alpha}
$$$$
+\left(\nabla_{\alpha} \nabla^{\mu} h-\nabla_{\beta} \nabla_{\alpha} h^{\mu \beta}-\nabla^{\beta} \nabla^{\mu} h_{\alpha \beta}+\square h_{\alpha}^{\mu}\right.
$$$$
\left.\left.+2\left(R_{\alpha \beta} h^{\mu \beta}+R^{\mu \beta} h_{\alpha \beta}\right)-R_{\alpha}^{\mu} h\right) \nabla^{\alpha} \xi^{\nu}\right]
$$$$
+\frac{\mathrm{Q} a \epsilon}{2 \mathrm{a}} \frac{\partial \mathrm{a}}{\partial \phi^{I}} \delta \phi^{I}-\Theta_{\mathrm{a}}^{\mu} \xi^{\nu}-[\mu \leftrightarrow \nu],
$$

$$
\begin{aligned}
& k_{\mathrm{b} \epsilon}^{\mu \nu}(\delta \Phi, \Phi)=\frac{\mathrm{b}}{8 \pi G}\left[\left(2\left(R_{\alpha \beta \gamma}^{\mu}-R_{\beta \alpha \gamma}^{\mu}\right) h^{\nu \gamma}+R_{\alpha \beta}^{\mu \nu} h\right.\right.
\end{aligned}
$$

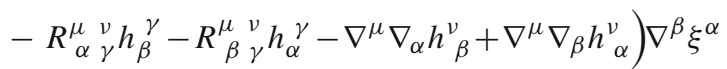

$$
\begin{aligned}
& +\left(R^{\mu \beta}\left(\nabla_{\beta} h_{\alpha}^{\nu}-\nabla_{\alpha} h_{\beta}^{v}\right)+R_{\beta \gamma}^{\mu \nu} \nabla^{\gamma} h_{\alpha}^{\beta}\right. \\
& +\frac{1}{2} R_{\alpha \gamma}^{\mu \nu}\left(\nabla_{\beta} h^{\beta \gamma}-\nabla^{\gamma} h\right) \\
& +2\left(\nabla_{\beta} R_{\alpha}^{\mu}-\nabla^{\mu} R_{\alpha \beta}\right) h^{\nu \beta}+\nabla^{\mu} \nabla_{\beta} \nabla_{\alpha} h^{\nu \beta} \\
& -\nabla^{\mu} \square h_{\alpha}^{v}+\nabla^{\mu} R_{\alpha}^{v} h+\nabla^{\mu} R_{\beta}^{v} h_{\alpha}^{\beta} \\
& \left.\left.-\nabla^{\mu}\left(R_{\beta \alpha \gamma}^{v} h^{\beta \gamma}\right)\right) 2 \xi^{\alpha}\right]+\frac{\mathrm{Q}_{\mathrm{b} \epsilon}^{\mu \nu}}{2 \mathrm{~b}} \frac{\partial \mathrm{b}}{\partial \phi^{I}} \delta \phi^{I}-\Theta_{\mathrm{b}}^{\mu} \xi^{\nu}-[\mu \leftrightarrow \nu],
\end{aligned}
$$

$$
\begin{aligned}
& k_{\mathrm{c} \epsilon}^{\mu \nu}(\delta \Phi, \Phi)=\frac{1}{8 \pi G}\left[\left(\frac{-h}{2} \mathrm{c}_{a b} F^{a \mu \nu}+2 \mathrm{c}_{a b} F^{a \mu \sigma} h_{\sigma}^{\nu}\right.\right. \\
& \left.-\mathrm{c}_{a b} \delta F^{a \mu \nu}-\frac{\partial \mathrm{c}_{a b}}{\partial \phi^{I}} F^{a \mu \nu} \delta \phi^{I}\right)\left(\xi^{\alpha} A_{\alpha}^{b}+\lambda^{b}\right) \\
& \left.-\mathrm{c}_{a b} F^{a \mu \nu} \xi^{\alpha} \delta A_{\alpha}^{b}-2 \mathrm{c}_{a b} F^{a \alpha \mu} \xi^{\nu} \delta A_{\alpha}^{b}\right]-[\mu \leftrightarrow \nu],
\end{aligned}
$$

$k_{\mathrm{d} \epsilon}^{\mu \nu}(\delta \Phi, \Phi)=\frac{1}{4 \pi G}\left[\xi^{\nu} \mathrm{d}_{I J} \nabla^{\mu} \phi^{I} \delta \phi^{J}\right]-[\mu \leftrightarrow \nu]$.

Having $\boldsymbol{k}_{\epsilon}$, and equipped with the parametric variations $\hat{\delta} \Phi$, calculation of the conserved charges associated with the exact symmetries $\eta=\left\{\zeta, \lambda^{a}\right\}$ of the (black hole) solutions $\hat{\Phi}\left(x^{\mu} ; p_{j}\right)$ to the Lagrangian (3.1) can be performed.

\section{Some examples}

To exemplify, in this section we will work out conserved charges and first law(s) of thermodynamics for some black hole solutions to the Lagrangian (3.1).

Example $1 z=3$ Lifshitz black hole in $d=3$

Consider

$f=R+\frac{13}{l^{2}}-\frac{3 l^{2}}{4} R^{2}, \quad \mathrm{a}=2 l^{2}, \quad \mathrm{~b}=\mathrm{c}=\mathrm{d}=0$,

i.e. the new massive gravity (NMG) Lagrangian [58]

$$
\mathcal{L}=\frac{1}{16 \pi G}\left(R-2 \Lambda+\frac{1}{\mathfrak{m}^{2}}\left(R_{\mu \nu} R^{\mu \nu}-\frac{3}{8} R^{2}\right)\right),
$$

in which $\Lambda=-\frac{13}{2 l^{2}}$ and $\mathfrak{m}^{2}=\frac{1}{2 l^{2}}$. We can have a family of black holes $\hat{g}_{\alpha \beta}\left(x^{\mu} ; m\right)$ as solution to this theory in the 3 -dimensional case $[59,60]$,

$$
\mathrm{d} s^{2}=-\left(\frac{r}{l}\right)^{2 z}\left(1-\frac{m l^{2}}{r^{2}}\right) \mathrm{d} t^{2}+\frac{\mathrm{d} r^{2}}{\frac{r^{2}}{l^{2}}\left(1-\frac{m l^{2}}{r^{2}}\right)}+r^{2} \mathrm{~d} \varphi^{2}
$$

for $z=3$. Let us analyze the thermodynamics of this family of black holes using SPSM. Putting Eq. (4.1) into the general result Eq. (3.12), $k_{\epsilon}^{\mu \nu}$ can be read for our specific theory. Then, choosing $\partial \Sigma$ to be surfaces of constant $(t, r)$ for simplicity, the conserved charge variations for an exact symmetry $\eta$ can be simply read through

$\hat{\delta} H_{\eta}=\oint_{\partial \Sigma} \boldsymbol{k}_{\eta}\left(\hat{\delta} g_{\alpha \beta}, \hat{g}_{\alpha \beta}\right)=\int_{0}^{2 \pi} \sqrt{-\hat{g}} k_{\eta}^{t r}\left(\hat{\delta} g_{\alpha \beta}, \hat{g}_{\alpha \beta}\right) \mathrm{d} \varphi$, 
in which $k_{\eta}^{t r}$ is the $t r$ component of the $k_{\eta}^{\mu \nu}$. Inserting parametric variations $\hat{\delta} g_{\alpha \beta}=\frac{\partial \hat{g}_{\alpha \beta}}{\partial m} \delta m$ in it, conserved charges can be calculated, irrespective of the asymptotic Lifshitz behavior.

Mass: We can choose the stationarity Killing $-\partial_{t}$ as the generator to which the mass is associated. The minus sign has been adopted to make the mass and entropy positive. Hence, by $\eta_{M}=\left\{-\partial_{t}, 0\right\}$ the result of calculating Eq. (4.4) is

$$
\hat{\delta} M \equiv \hat{\delta} H_{\eta_{M}}=\frac{m}{2 G} \delta m=\hat{\delta}\left(\frac{m^{2}}{4 G}\right) \quad \Rightarrow \quad M=\frac{m^{2}}{4 G}
$$

The reference point (constant of integration) was chosen $M=$ 0 for the geometry with $m=0$.

Angular momentum: Choosing $\eta_{J}=\left\{-\partial_{\varphi}, 0\right\}$, and by a similar analysis as the mass, angular momentum turns out to be

$\hat{\delta} J \equiv \hat{\delta} H_{\eta_{J}}=0 \times \delta m \quad \Rightarrow \quad J=0$.

Entropy: The surface gravity on the horizon of this solution is $\kappa_{\mathrm{H}}=\frac{r_{\mathrm{H}}^{3}}{l^{4}}$ in which $r_{\mathrm{H}}=\sqrt{m l^{2}}$. The entropy of the horizon is defined to be the conserved charge associated with the horizon Killing vector $\zeta_{\mathrm{H}}$ normalized by the Hawking temperature $T_{\mathrm{H}}=\frac{\kappa_{\mathrm{H}}}{2 \pi}$. Therefore, by $\eta_{\mathrm{H}}=\frac{2 \pi}{\kappa_{\mathrm{H}}}\left\{\zeta_{\mathrm{H}}, 0\right\}$ and the identity $\zeta_{\mathrm{H}}=-\partial_{t}$, the entropy attributed to the horizon, via a similar integration to the other conserved charges, is calculated to be

$\hat{\delta} S_{\mathrm{H}} \equiv \hat{\delta} H_{\eta_{\mathrm{H}}}=\frac{\pi l}{G \sqrt{m}} \delta m=\hat{\delta}\left(\frac{2 \pi r_{\mathrm{H}}}{G}\right) \Rightarrow S_{\mathrm{H}}=\frac{2 \pi r_{\mathrm{H}}}{G}$.

The reference point is chosen to be $S_{\mathrm{H}}=0$ for the geometry identified by $m=0$. Notice that the entropy is proportional to the area (here the length) of the horizon, but without the usual factor of $\frac{1}{4}$. The results above are in agreement with the results reported in Refs. [35,61].

First law: Having made the entropy free of being calculated on the horizons, the first law of thermodynamics would follow:

$\eta_{\mathrm{H}}=\frac{1}{T_{\mathrm{H}}} \eta_{M} \stackrel{\text { linearity of } \delta H_{\epsilon} \mathrm{in} \epsilon}{\longrightarrow} \quad \delta S_{\mathrm{H}}=\frac{1}{T_{\mathrm{H}}} \delta M$.

Although $\delta$ in the equation above is a generic perturbation which satisfies linearized EoM, but it can be cross-checked for the parametric variations using the explicit results for $\hat{\delta} M$ and $\hat{\delta} S_{\mathrm{H}}$ in Eqs. (4.5) and (4.7).

\section{Example 2 Warped $\mathrm{AdS}_{3}$}

A warped $\mathrm{AdS}_{3}$ is a 3-dimensional black hole identified by two parameters $p_{1}=m$ and $p_{2}=j$ :

$$
\begin{aligned}
\mathrm{d} s^{2} & =\left(\frac{-r^{2}}{l^{2}}+8\left(m-\frac{j}{l}\right)\right) \mathrm{d} t^{2}+\frac{\mathrm{d} r^{2}}{\frac{16 j^{2}}{r^{2}}+\frac{r^{2}}{l^{2}}-8\left(m-\frac{j}{l}\right)} \\
+r^{2} \mathrm{~d} \varphi^{2}-\left(\omega_{t} \mathrm{~d} t-\omega_{\varphi} \mathrm{d} \varphi\right)^{2} & \\
\omega_{t} & \equiv \frac{H\left(-r^{2}+8 l^{2} m-4 l j\right)}{2 l^{2} \sqrt{m}}, \quad \omega_{\varphi} \equiv \frac{H\left(r^{2}+4 l j\right)}{2 l \sqrt{m}} .
\end{aligned}
$$

It is a solution $[62,63]$ to the NMG theory, described by the Lagrangian in Eq. (4.2) with

$$
\Lambda=\frac{84 H^{4}+60 H^{2}-35}{2 l^{2}\left(17-42 H^{2}\right)}, \quad \frac{1}{\mathfrak{m}^{2}}=\frac{2 l^{2}}{42 H^{2}-17} .
$$

After extracting $k_{\epsilon}^{\mu \nu}$ for this theory from the general result in Eq. (3.12), and equipped with the parametric variations

$\hat{\delta} g_{\alpha \beta}=\frac{\partial \hat{g}_{\alpha \beta}}{\partial m} \delta m+\frac{\partial \hat{g}_{\alpha \beta}}{\partial j} \delta j$,

one can find the conserved charges by an integration similar to the Eq. (4.4). Notice that because of the linearity of $\delta H_{\eta}(\delta \Phi, \Phi)$ in $\delta \Phi$, the parametric variations can be inserted term by term into the calculations. This makes the calculations to be performed easier.

Mass: By $\eta_{M}=\left\{\partial_{t}+\Omega_{\infty} \partial_{\varphi}, 0\right\}$ in which $\Omega_{\infty}=\frac{-1}{l}$, it turns out that

$$
\begin{aligned}
\hat{\delta} M & \equiv \hat{\delta} H_{\eta_{M}}=\frac{16\left(1-2 H^{2}\right)^{\frac{3}{2}}}{G\left(17-42 H^{2}\right)} \delta m+0 \times \delta j \\
& \Rightarrow M=\frac{16\left(1-2 H^{2}\right)^{\frac{3}{2}} m}{G\left(17-42 H^{2}\right)} .
\end{aligned}
$$

Angular momentum: Choosing $\eta_{J}=\left\{-\partial_{\varphi}, 0\right\}$,

$$
\begin{aligned}
\hat{\delta} J & \equiv \hat{\delta} H_{\eta_{J}}=0 \times \delta m+\frac{16\left(1-2 H^{2}\right)^{\frac{3}{2}}}{G\left(17-42 H^{2}\right)} \delta j \\
& \Rightarrow J=\frac{16\left(1-2 H^{2}\right)^{\frac{3}{2}} j}{G\left(17-42 H^{2}\right)} .
\end{aligned}
$$

Entropies: There are two horizons in the warped $\mathrm{AdS}_{3}$ geometry (4.9). So, we would find two entropies attributed to them. The horizons are situated at $r_{ \pm}^{2}=4 l^{2}\left(m-\frac{j}{l} \pm \sqrt{m\left(m-\frac{2 j}{l}\right)}\right.$, collectively denoted by $r_{\mathrm{H}}$. The surface gravities, angular velocities, and the Killing vectors of the horizons are

$\kappa_{\mathrm{H}}=\frac{r_{\mathrm{H}}^{4}-16 l^{2} j^{2}}{l^{2} r_{\mathrm{H}}^{3}}, \quad \Omega_{\mathrm{H}}=\frac{4 j}{r_{\mathrm{H}}^{2}}, \quad \zeta_{\mathrm{H}}=\partial_{t}+\Omega_{\mathrm{H}} \partial_{\varphi}$,

respectively. Integrating over arbitrary surfaces of constant time and radius, the entropies as conserved charges associated with the exact symmetries $\eta_{\mathrm{H}}=\frac{2 \pi}{\kappa_{\mathrm{H}}}\left\{\zeta_{\mathrm{H}}, 0\right\}$ are calculated to be 


$$
\begin{aligned}
\hat{\delta} S_{\mathrm{H}} & =\frac{\partial\left(\frac{8 \pi\left(1-2 H^{2}\right)^{\frac{3}{2}} r_{\mathrm{H}}}{G\left(17-42 H^{2}\right)}\right)}{\partial m} \delta m+\frac{\partial\left(\frac{8 \pi\left(1-2 H^{2}\right)^{\frac{3}{2}} r_{\mathrm{H}}}{G\left(17-42 H^{2}\right)}\right)}{\partial j} \delta j \\
& \Rightarrow S_{\mathrm{H}}=\frac{8 \pi\left(1-2 H^{2}\right)^{\frac{3}{2}} r_{\mathrm{H}}}{G\left(17-42 H^{2}\right)} .
\end{aligned}
$$

The reference point for all of the charges above have been chosen to vanish on the geometry identified by $m=j=0$. Our results would match exactly with the results reported in Refs. [64,65] if one replaces the parameter $m \rightarrow m-\frac{j}{l}$. The difference originates from considering the asymptotic angular velocity $\Omega_{\infty}$ in the definition of mass. Hence, the mass calculated here is different from the mass reported in $[64,65]$ by a term, which is $\Omega_{\infty} J$.

First laws: For any generic perturbation which satisfies the linearized EoM, the first laws follow:

$$
\begin{aligned}
& \eta_{\mathrm{H}}=\frac{1}{T_{\mathrm{H}}}\left(\eta_{M}-\left(\Omega_{\mathrm{H}}-\Omega_{\infty}\right) \eta_{J}\right) \stackrel{\text { linearity of } \delta H_{\epsilon} \text { in } \epsilon}{\longrightarrow} \\
& \delta S_{\mathrm{H}}=\frac{1}{T_{\mathrm{H}}}\left(\delta M-\left(\Omega_{\mathrm{H}}-\Omega_{\infty}\right) \delta J\right),
\end{aligned}
$$

which can be checked for the parametric variations in (4.12), (4.13), and (4.15).

Example 3 Schwarzschild-AdS black holes in $d$ dimensions

The family of black holes

$$
\mathrm{d} s^{2}=-\left(1-\frac{2 G m}{r^{d-3}}+\frac{r^{2}}{l^{2}}\right) \mathrm{d} t^{2}+\frac{\mathrm{d} r^{2}}{1-\frac{2 G m}{r^{d-3}}+\frac{r^{2}}{l^{2}}}+r^{2} \mathrm{~d} \Omega_{d-2}^{2}
$$

are solutions to the theories

$$
\mathcal{L}=\frac{1}{16 \pi G}\left(R-2 \Lambda+\alpha R^{2}+\mathrm{a} R_{\mu \nu} R^{\mu \nu}\right),
$$

where $\alpha$ and a are arbitrary constants, and $\Lambda=\frac{-l^{2}\left(d^{2}-3 d+2\right)+(\alpha d+\mathrm{a})(d-4)(d-1)^{2}}{2 l^{4}}$. For these theories, $k_{\epsilon}^{\mu \nu}$ can be read through the general result (3.12) by putting $f=R-2 \Lambda+\alpha R^{2}$, the arbitrary constant factor a, and vanishing $\mathrm{b}=\mathrm{c}_{a b}=\mathrm{d}_{I J}=0$. Similar to the previous examples, one can choose $\partial \Sigma$ to be surfaces of constant $(t, r)$ for simplicity. Hence, the conserved charge variations for an exact symmetry $\eta$ would be

$\hat{\delta} H_{\eta}=\int_{S_{d-2}} \sqrt{-\hat{g}} k_{\eta}^{t r}\left(\hat{\delta} g_{\alpha \beta}, \hat{g}_{\alpha \beta}\right)$.

The integration is taken over the $d$-2-dimensional spheres, e.g. in four dimensions it is $\int_{0}^{\pi} \int_{0}^{2 \pi} \mathrm{d} \theta \mathrm{d} \varphi$. By parametric variations $\hat{\delta} g_{\alpha \beta}=\frac{\partial \hat{g}_{\alpha \beta}}{\partial m} \delta m$, the conserved charges can be calculated.
Mass: For the exact symmetry $\eta_{M}=\left\{\partial_{t}, 0\right\}$, the result of calculating Eq. (4.19) is

$\hat{\delta} M=\mathcal{X} \times \frac{(d-2) \Omega_{d-2}}{8 \pi} \delta m \Rightarrow M=\mathcal{X} \times \frac{(d-2) \Omega_{d-2}}{8 \pi} m$,

where

$$
\mathcal{X}=\frac{l^{2}-2 d(d-1) \alpha-2(d-1) \mathrm{a}}{l^{2}}, \quad \Omega_{d-2}=\frac{2 \pi^{\frac{d-1}{2}}}{\Gamma\left(\frac{d-1}{2}\right)} .
$$

The reference point has been chosen to be $M=0$ for the geometry which is identified by $m=0$.

Angular momentum: By $\eta_{J}=\left\{-\partial_{\varphi}, 0\right\}$, angular momentum is calculated to be

$\hat{\delta} J=0 \times \delta m \quad \Rightarrow \quad J=0$.

Entropy: Surface gravity is a property of solutions, and it is independent of the theory. For the event horizon of the solutions (4.17), it is

$\kappa_{\mathrm{H}}=\frac{(d-1) r_{\mathrm{H}}^{d-2}+(d-3) l^{2} r_{\mathrm{H}}^{d-4}}{2 l^{2} r_{\mathrm{H}}^{d-3}}$,

in which $r_{\mathrm{H}}$ solves the equation $r_{\mathrm{H}}^{d-1}+l^{2} r_{\mathrm{H}}^{d-3}-2 G m l^{2}=0$. By $\eta_{\mathrm{H}}=\frac{2 \pi}{\kappa_{\mathrm{H}}}\left\{\zeta_{\mathrm{H}}, 0\right\}$ in which $\zeta_{\mathrm{H}}=\partial_{t}$, the entropy variation attributed to the event horizon, which is calculated on arbitrary surfaces of integration, would be

$\hat{\delta} S_{\mathrm{H}}=\mathcal{X} \times \frac{(d-2) \Omega_{d-2}}{4 \kappa_{\mathrm{H}}} \delta m$.

Noticing the linearity of $\delta H_{\epsilon}$ (2.9) in $\epsilon$, this result can also be found by multiplication of $\hat{\delta} M$, which is calculated in Eq. (4.20), by the factor $\frac{2 \pi}{\kappa_{\mathrm{H}}}$. Hence, using $\frac{\partial r_{\mathrm{H}}}{\partial m}=$ $\frac{2 G l^{2}}{(d-1) r_{\mathrm{H}}^{d-2}+(d-3) l^{2} r_{\mathrm{H}}^{d-4}}$

$$
\begin{aligned}
\hat{\delta} S_{\mathrm{H}} & =\frac{\partial\left(\frac{\mathcal{X} \Omega_{d-2} r_{\mathrm{H}}^{d-2}}{4 G}\right)}{\partial r_{\mathrm{H}}} \frac{\partial r_{\mathrm{H}}}{\partial m} \delta m=\hat{\delta}\left(\mathcal{X} \times \frac{\Omega_{d-2} r_{\mathrm{H}}^{d-2}}{4 G}\right) \\
& \Rightarrow S_{\mathrm{H}}=\mathcal{X} \times \frac{\Omega_{d-2} r_{\mathrm{H}}^{d-2}}{4 G} .
\end{aligned}
$$

First law: It is simply

$$
\eta_{\mathrm{H}}=\frac{1}{T_{\mathrm{H}}} \eta_{M} \stackrel{\text { linearity of } \delta H_{\epsilon} \text { in } \epsilon}{\longrightarrow} \quad \delta S_{\mathrm{H}}=\frac{1}{T_{\mathrm{H}}} \delta M,
$$


which can be checked for the parametric variations by the results in Eqs. (4.20) and (4.24). We finish this example by mentioning two remarks:

- One can add any factor of Gauss-Bonnet Lagrangian $\mathcal{L}_{\mathrm{GB}} \propto R^{2}-4 R_{\mu \nu} R^{\mu \nu}+R_{\mu \nu \alpha \beta} R^{\mu \nu \alpha \beta}$ in $d=3,4$ to the Lagrangian (4.18), without affecting the EoM and conserved charges.

- The family of black holes in this example has the property $R_{\mu \nu}=\frac{R}{d} g_{\mu \nu}$. The geometries with such a property are called Einstein geometries. The following theorem (see Ref. [66] and references therein) sheds light on the results of the calculations above.

Theorem: Any theory which is described by a Lagrangian $\mathcal{L}=\mathcal{L}\left(g_{\alpha \beta}, R_{\mu \nu}\right)$, with an Einstein geometry $g_{\alpha \beta}$ as a solution, can be mapped to the Einstein-Hilbert theory with the solution

$$
\bar{g}_{\alpha \beta}=\mathcal{X}^{\frac{2}{d-2}} g_{\alpha \beta}, \quad \mathcal{X}=\left[\frac{d}{2 R} \times \mathcal{L}\right]_{\text {on-shell }} .
$$

This theorem clarifies the observation that the mass and entropy calculated above are the mass and entropy in the Einstein-Hilbert theory multiplied by the factor $\mathcal{X}$.

Example 4 Charged static BTZ black hole Our last example, although it is in the context of the Lagrangian (3.1), but does not have higher curvature terms. It would be a pedagogical example in the presence of the gauge fields. Moreover, it remedies the divergent results appearing in the literature. This last example is the electrically charged static BTZ black hole $[67,68]$

$$
\begin{aligned}
\mathrm{d} s^{2}= & -\left(-G m+\frac{r^{2}}{l^{2}}-\frac{q^{2}}{2} \log \frac{r}{l}\right) \mathrm{d} t^{2} \\
& +\frac{\mathrm{d} r^{2}}{-G m+\frac{r^{2}}{l^{2}}-\frac{q^{2}}{2} \log \frac{r}{l}}+r^{2} \mathrm{~d} \varphi^{2} \\
\hat{A}= & -\frac{q}{2} \log \left(\frac{r}{l}\right) \mathrm{d} t
\end{aligned}
$$

as a solution to the theory described by

$\mathcal{L}=\frac{1}{16 \pi G}\left(R-2 \Lambda-F_{\mu \nu} F^{\mu \nu}\right)$

for $\Lambda=\frac{-1}{l^{2}} \cdot k_{\epsilon}^{\mu \nu}$ for this theory can be read through Eq. (3.12) by putting $f=R-2 \Lambda, \mathrm{a}=\mathrm{b}=\mathrm{d}_{I J}=0$, and $\mathrm{c}_{a b}=\delta_{a b}$. Making the simplifying choice of taking $\partial \Sigma$ to be the lines of constant $(t, r)$, conserved charge variations for an exact symmetry $\eta$ would be

$\hat{\delta} H_{\eta}=\int_{0}^{2 \pi} \sqrt{-\hat{g}} k_{\eta}^{t r}(\hat{\delta} \Phi, \hat{\Phi}) \mathrm{d} \varphi$.

The dynamical fields $\hat{\Phi}$ are the metric $\hat{g}_{\alpha \beta}$ and gauge field $\hat{A}$ in Eq. (4.28), parametrized by $p_{j}=\{m, q\}$. So, the parametric variations would be $\hat{\delta} g_{\alpha \beta}=\frac{\partial \hat{g}_{\alpha \beta}}{\partial m} \delta m+\frac{\partial \hat{g}_{\alpha \beta}}{\partial q} \delta q, \quad \hat{\delta} A_{\mu}=\frac{\partial \hat{A}_{\mu}}{\partial m} \delta m+\frac{\partial \hat{A}_{\mu}}{\partial q} \delta q$.

Mass: In the specific chosen gauge for $\hat{A}$ in Eq. (4.28), $\Phi_{\infty}=0$. By $\eta_{M}=\left\{\partial_{t},-\Phi_{\infty}\right\}$, the Eq. (4.30) yields

$\hat{\delta} M=\frac{1}{8} \times \delta m+0 \times \delta q \quad \Rightarrow \quad M=\frac{m}{8}$.

Angular momentum: For $\eta_{J}=\left\{-\partial_{\varphi}, 0\right\}$,

$\hat{\delta} J=0 \times \delta m+0 \times \delta q \quad \Rightarrow \quad J=0$.

Electric charge: For the exact symmetry $\eta_{Q}=\{0,1\}$,

$\hat{\delta} Q=0 \times \delta m+\frac{1}{4 G} \times \delta q \quad \Rightarrow \quad Q=\frac{q}{4 G}$.

Entropies: For any horizon present in this geometry, one can associate an entropy. Surface gravities, electric potentials, and horizon Killing vectors for different horizons would be collectively

$\kappa_{\mathrm{H}}=\frac{r_{\mathrm{H}}}{l^{2}}-\frac{q^{2}}{4 r_{\mathrm{H}}}, \quad \Phi_{\mathrm{H}}=-\frac{q}{2} \log \left(\frac{r_{\mathrm{H}}}{l}\right), \quad \zeta_{\mathrm{H}}=\partial_{t}$,

where $r_{\mathrm{H}}$ denotes the radius of any one of the horizons. By the choice of $\eta_{\mathrm{H}}=\frac{2 \pi}{\kappa_{\mathrm{H}}}\left\{\zeta_{\mathrm{H}},-\Phi_{\mathrm{H}}\right\}$,

$\hat{\delta} S_{\mathrm{H}}=\frac{2 \pi}{\kappa_{\mathrm{H}}}\left(\frac{1}{8} \delta m-\frac{\Phi_{\mathrm{H}}}{4 G} \delta q\right)$.

Now, by inserting the relations

$$
\frac{\partial r_{\mathrm{H}}}{\partial m}=\frac{G}{\frac{2 r_{\mathrm{H}}}{l^{2}}-\frac{q^{2}}{2 r_{\mathrm{H}}}}, \quad \frac{\partial r_{\mathrm{H}}}{\partial q}=\frac{q \log \left(\frac{r_{\mathrm{H}}}{l}\right)}{\frac{2 r_{\mathrm{H}}}{l^{2}}-\frac{q^{2}}{2 r_{\mathrm{H}}}},
$$

we find

$$
\begin{aligned}
\hat{\delta} S_{\mathrm{H}} & =\frac{2 \pi}{4 G}\left(\frac{\partial r_{\mathrm{H}}}{\partial m} \delta m+\frac{\partial r_{\mathrm{H}}}{\partial q} \delta q\right)=\hat{\delta}\left(\frac{2 \pi r_{\mathrm{H}}}{4 G}\right) \\
& \Rightarrow S_{\mathrm{H}}=\frac{2 \pi r_{\mathrm{H}}}{4 G} .
\end{aligned}
$$

Reference points for the charges above are chosen to vanish for the pure $\mathrm{AdS}_{3}$ geometry, i.e. the geometry identified by $m=q=0$. In comparison with the calculations done in the literature (see e.g. Ref.[68]), the charges above are finite, and one does not need to regularize any divergent result. Notice that by replacing $\log \left(\frac{r}{l}\right) \rightarrow \log \left(\frac{r}{r_{0}}\right)$ for some $r_{0}$, the solution would remain a solution, but $M \rightarrow M+\frac{q^{2}}{16 G} \log \left(\frac{l}{r_{0}}\right)$. 
First laws: For any one of the horizons, the first law would be

$$
\begin{aligned}
& \eta_{\mathrm{H}}=\frac{1}{T_{\mathrm{H}}}\left(\eta_{M}-\left(\Phi_{\mathrm{H}}-\Phi_{\infty}\right) \eta_{Q}\right) \stackrel{\text { linearity of } \delta H_{\epsilon} \text { in } \epsilon}{\longrightarrow} \\
& \delta S_{\mathrm{H}}=\frac{1}{T_{\mathrm{H}}}\left(\delta M-\left(\Phi_{\mathrm{H}}-\Phi_{\infty}\right) \delta Q\right),
\end{aligned}
$$

which can be checked for the parametric variations via Eqs. (4.32), (4.34), and (4.36).

At the end, it is worth mentioning that in the definition of $\eta_{\mathrm{H}}$ in the examples above, we tacitly assumed $\kappa_{\mathrm{H}} \neq 0$. For $\kappa_{\mathrm{H}}=0$ cases, which are called extremal black holes, one can find an infinite number of exact symmetries in their near horizon regions, as generators of the entropy $[29,49]$. Using any one of these generators, the SPSM would reproduce the entropy for the extremal black holes too. An explicit example for such an analysis can be found in Ref. [31], where the near horizon of the extremal Kerr-Newman black hole is studied.

\section{Conclusion}

In this work, after reviewing the solution phase space method, we applied it to a family of higher curvature gravitational theories. The family which we focused on, contained $f(R)$ gravity, quadratic Riemann and Ricci terms, an arbitrary number of Abelian gauge fields, and arbitrary scalar fields. After elaborating the $\boldsymbol{k}_{\epsilon}$, which is pragmatically the most important differential form for the calculations, four families of black hole solutions were analyzed. Specifically, their conserved charges were calculated, confirming the results formerly calculated by the other methods. By the way, the results ameliorated the divergence appearing in the calculation of mass for the charged static BTZ black hole. The main advantages of the method are: (1) it works for any higher curvature theory in any dimension, (2) asymptotics and horizons are unimportant in the charge calculations, (3) conserved charges are automatically regular, (4) conserved charges are unambiguous, (5) all the charges, including the entropy and electric charge, are calculated by a single machinery, (6) the proof of the first law(s) is very simple.

Acknowledgements K.H would like to thank members of the quantum gravity group at IPM, specifically M.M. Sheikh-Jabbari and M.H. Vahidinia, for useful discussions. He also thanks ICTP for its hospitality during the Workshop on Topics in Three Dimensional Gravity. Some of the calculations in this paper have been done using the HPC cluster in IPM. This work has been supported by the Allameh Tabatabaii Prize Grant of National Elites Foundation of Iran and the Saramadan grant of the Iranian vice presidency in science and technology.

Open Access This article is distributed under the terms of the Creative Commons Attribution 4.0 International License (http://creativecomm ons.org/licenses/by/4.0/), which permits unrestricted use, distribution, and reproduction in any medium, provided you give appropriate credit to the original author(s) and the source, provide a link to the Creative
Commons license, and indicate if changes were made. Funded by SCOAP ${ }^{3}$.

\section{References}

1. L.B. Szabados, Quasi-local energy-momentum and angular momentum in GR: a review article. Living Rev. Rel. 7, 4 (2004)

2. M. Bañados, I.A. Reyes, A short review on Noether's theorems, gauge symmetries and boundary terms, for students (2016). arXiv: 1601.03616

3. A. Komar, Covariant conservation laws in general relativity. Phys. Rev. 113, 934-936 (1959)

4. R.L. Arnowitt, S. Deser, C.W. Misner, Dynamical structure and definition of energy in general relativity. Phys. Rev. 116, 13221330 (1959)

5. R.L. Arnowitt, S. Deser, C.W. Misner, Canonical variables for general relativity. Phys. Rev. 117, 1595-1602 (1960)

6. R.L. Arnowitt, S. Deser, C.W. Misner, The dynamics of general relativity. Gen. Rel. Grav. 40, 1997-2027 (2008). arXiv:gr-qc/0405109

7. E. Gourgoulhon, $3+1$ formalism and bases of numerical relativity (2007). arXiv:gr-qc/0703035

8. H. Bondi, M.G.J. van der Burg, A.W.K. Metzner, Gravitational waves in general relativity. 7. Waves from axisymmetric isolated systems. Proc. Roy. Soc. Lond. A 269, 21-52 (1962)

9. R. Sachs, Asymptotic symmetries in gravitational theory. Phys. Rev. 128, 2851-2864 (1962)

10. T. Regge, C. Teitelboim, Role of surface integrals in the Hamiltonian formulation of general relativity. Ann. Phys. 88, 286 (1974)

11. S. Hollands, A. Ishibashi, D. Marolf, Comparison between various notions of conserved charges in asymptotically AdSspacetimes. Class. Quantum Grav. 22, 2881-2920 (2005). arXiv:hep-th/0503045

12. J.D. Brown, J.W. York Jr., Quasilocal energy and conserved charges derived from the gravitational action. Phys. Rev. D 47, 1407-1419 (1993). arXiv:gr-qc/9209012

13. V. Balasubramanian, P. Kraus, A stress tensor for anti-de Sitter gravity. Commun. Math. Phys. 208, 413-428 (1999). arXiv:hep-th/9902121

14. L.F. Abbott, S. Deser, Stability of gravity with a cosmological constant. Nucl. Phys. B 195, 76-96 (1982)

15. S. Deser, B. Tekin, Gravitational energy in quadratic curvature gravities. Phys. Rev. Lett. 89, 101101 (2002). arXiv:hep-th/0205318

16. S. Deser, B. Tekin, Energy in generic higher curvature gravity theories, Phys. Rev. D 67, 084009 (2003). arXiv:hep-th/0212292

17. W. Kim, S. Kulkarni, S.-H. Yi, Quasilocal conserved charges in a covariant theory of gravity. Phys. Rev. Lett. 111(8), 081101 (2013). [Erratum: Phys. Rev. Lett. 112, no. 7, 079902 (2014)]. arXiv: 1306.2138

18. R.M. Wald, A. Zoupas, A general definition of 'conserved quantities' in general relativity and other theories of gravity. Phys. Rev. D 61, 084027 (2000). arXiv:gr-qc/9911095

19. G. Barnich, Boundary charges in gauge theories: using Stokes theorem in the bulk. Class. Quantum Grav. 20, 3685-3698 (2003). arXiv:hep-th/0301039

20. A. Ashtekar, L. Bombelli, R. Koul, Phase space formulation of general relativity without a $3+1$ splitting. in Lect. Notes Phys., vol. 278 (1987), pp. 356-359

21. A. Ashtekar, L. Bombelli, O. Reula, The covariant phase space of asymptotically flat gravitational fields. in Mechanics, analysis and geometry: 200 years after Lagrange, ed. by M. Francaviglia (1990), pp. 417-450 
22. C. Crnkovic, , E. Witten, Covariant description of canonical formalism in geometrical theories, in Three hundred years of gravitation, ed. by S.W. Hawking, W. Israel. (1987), pp. 676-684

23. J. Lee, R.M. Wald, Local symmetries and constraints. J. Math. Phys. 31, 725-743 (1990)

24. R.M. Wald, Black hole entropy is the Noether charge. Phys. Rev. D. 48, 3427-3431 (1993). arXiv:gr-qc/9307038

25. V. Iyer, R.M. Wald, Some properties of Noether charge and a proposal for dynamical black hole entropy. Phys. Rev. D, 50, 846-864 (1994). arXiv:gr-qc/9403028

26. K. Hajian, On Thermodynamics and phase space of near horizon extremal geometries, Ph.D. thesis (2015). arXiv:1508.03494

27. A. Seraj, Conserved charges, surface degrees of freedom, and black hole entropy, Ph.D thesis (2016). arXiv: 1603.02442

28. A. Corichi, I. Rubalcava-Garca, T. Vukasinac, Actions, topological terms and boundaries in first-order gravity: a review. Int. J. Mod. Phys. D 25(04), 1630011 (2016). arXiv:1604.07764

29. K. Hajian, A. Seraj, M.M. Sheikh-Jabbari, NHEG mechanics: laws of near horizon extremal geometry (Thermo) dynamics. JHEP. 1403, 014 (2014). arXiv: 1310.3727

30. G. Barnich, F. Brandt, Covariant theory of asymptotic symmetries, conservation laws and central charges. Nucl. Phys. B 633, 3-82 (2002). arXiv:hep-th/0111246

31. K. Hajian, M.M. Sheikh-Jabbari, Solution phase space and conserved charges: a general formulation for charges associated with exact symmetries. Phys. Rev. D 93(4), 044074 (2016). arXiv: 1512.05584

32. S. Nam, J.-D. Park, S.-H. Yi, Mass and angular momentum of black holes in new massive gravity. Phys. Rev. D 82, 124049 (2010). arXiv: 1009.1962

33. D.O. Devecioglu, O. Sarioglu, Conserved Killing charges of quadratic curvature gravity theories in arbitrary backgrounds. Phys. Rev. D 83, 021503 (2011). arXiv:1010.1711

34. G. Alkac, D.O. Devecioglu, Covariant symplectic structure and conserved charges of new massive gravity. Phys. Rev. D 85, 064048 (2012). arXiv:1202.1905

35. Y. Gim, W. Kim, S.-H. Yi, The first law of thermodynamics in Lifshitz black holes revisited. JHEP 07, 002 (2014). arXiv:1403.4704

36. S. Hyun, J. Jeong, S. Park, S. Yi, Quasilocal conserved charges and holography. Phys. Rev. D. 90(10), 104016 (2014). arXiv:1406.7101

37. J. Peng, Conserved charges of black holes in Weyl and EinsteinGauss-Bonnet gravities. Eur. Phys. J. C 74, 113156 (2014). arXiv: 1407.4875

38. Z.Y. Fan, H. Lu, Thermodynamical first laws of black holes in quadratically-extended gravities. Phys. Rev. D 91(6), 064009 (2015). arXiv:1501.00006

39. M. Bravo-Gaete, M. Hassaine, Thermodynamics of charged Lifshitz black holes with quadratic corrections. Phys. Rev. D 91(6), 064038 (2015). arXiv:1501.03348

40. M.R. Setare, H. Adami, Black hole conserved charges in generalized minimal massive gravity. Phys. Lett. B 744, 280-283 (2015). arXiv: 1504.01660

41. M.R. Setare, H. Adami, Entropy formula of black holes in minimal massive gravity and its application for BTZ black holes. Phys. Rev. D 91(10), 104039 (2015). arXiv: 1501.00920

42. M.R. Setare, H. Adami, Black hole entropy in the ChernSimonslike theories of gravity and Lorentz-diffeomorphism Noether charge. Nucl. Phys. B 902, 115 (2016). arXiv:1509.05972

43. Y. Myung, Phase transitions of the BTZ black hole in new massive gravity. Adv. High Energy Phys. 2015, 478273 (2015). arXiv: 1510.02853

44. K. Hajian, Conserved charges and first law of thermodynamics for Kerr-de Sitter black holes. Gen. Rel. Grav. 48(8), 114 (2016). arXiv:1602.05575
45. G. Compere, P.-J. Mao, A. Seraj, M.M. Sheikh-Jabbari, Symplectic and Killing symmetries of $\mathrm{AdS}_{3}$ gravity: holographic vs boundary gravitons. JHEP 01, 080 (2016). arXiv: 1511.06079

46. G. Compere, K. Hajian, A. Seraj, M.M. Sheikh-Jabbari, Extremal rotating black holes in the near-horizon limit: phase space and symmetry algebra. Phys. Lett. B 749, $443-447$ (2015). arXiv:1503.07861

47. G. Compere, K. Hajian, A. Seraj, M.M. Sheikh-Jabbari, Wiggling throat of extremal black holes. JHEP 10, 093 (2015). arXiv: 1506.07181

48. M.M. Sheikh-Jabbari, Residual diffeomorphisms and symplectic hair on black holes (2016). arXiv:1603.07862

49. K. Hajian, A. Seraj, M.M. Sheikh-Jabbari, Near horizon extremal geometry perturbations: dynamical field perturbations vs. parametric variations. JHEP, 1410, 111 (2014). arXiv:1407.1992

50. J.D. Bekenstein, Black holes and entropy. Phys. Rev. D 7, 2333 2346 (1973)

51. J.M. Bardeen, B. Carter, S.W. Hawking, The four laws of black hole mechanics. Commun. Math. Phys. 31, 161-170 (1973)

52. S.W. Hawking, Particle creation by black holes. Commun. Math. Phys. 43, 199-220 (1975)

53. C. Lanczos, Z. Phys. 73, 147 (1932)

54. C. Lanczos. Ann. Math. 39, 842 (1938)

55. D. Lovelock, J. Math. Phys. 12, 498 (1971)

56. T. Padmanabhan, D. Kothawala, Lanczos-Lovelock models of gravity. Phys. Rept. 531, 115 (2013). arXiv:1302.2151

57. K.S. Stelle, Classical gravity with higher derivatives. Gen. Rel. Grav. 9, 353-371 (1978)

58. E.A. Bergshoeff, O. Hohm, P.K. Townsend, Massive gravity in three dimensions. Phys. Rev. Lett. 102, 201301(2009). arXiv:0901.1766

59. E. Ayon-Beato, A. Garbarz, G. Giribet, M. Hassaine, Lifshitz black hole in three dimensions. Phys. Rev. D 80, 104029 (2009). arXiv:0909.1347

60. E. Ayon-Beato, A. Garbarz, G. Giribet, M. Hassaine, Analytic Lifshitz black holes in higher dimensions. JHEP 04, 030 (2010). arXiv: 1001.2361

61. O. Hohm, E. Tonni, A boundary stress tensor for higher-derivative gravity in AdS and Lifshitz backgrounds. JHEP, 1004, 093 (2010). arXiv: 1001.3598

62. G. Clement, Warped AdS(3) black holes in new massive gravity. Class. Quantum Grav. 26, 105015 (2009). arXiv:0902.4634

63. S. Detournay, C. Zwikel, Phase transitions in warped $\mathrm{AdS}_{3}$ gravity. JHEP 1505, 074 (2015). arXiv:1504.00827

64. M. Ghodrati, A. Naseh, Phase transitions in BHT Massive Gravity. arXiv: 1601.04403

65. S. Detournay, L.A. Douxchamps, G.S. Ng, C. Zwikel, Warped $\mathrm{AdS}_{3}$ black holes in higher derivative gravity theories. arXiv: 1602.09089

66. M.R.M. Mozaffar, A. Mollabashi, M.M. Sheikh-Jabbari, M.H. Vahidinia, Holographic entanglement entropy, field redefinition invariance and higher derivative gravity theories. arXiv:1603.05713

67. M. Banados, C. Teitelboim, J. Zanelli, The Black hole in three-dimensional space-time. Phys. Rev. Lett. 69, 1849 (1992). arXiv:hep-th/9204099

68. C. Martinez, C. Teitelboim, J. Zanelli, Charged rotating black hole in three space-time dimensions. Phys. Rev. D 61, 104013 (2000). arXiv:hep-th/9912259 